\title{
Efficient isolation of specific genomic regions retaining molecular interactions by the iChIP system using recombinant exogenous DNA-binding proteins
}

Toshitsugu Fujita and Hodaka Fujii*

\begin{abstract}
Background: Comprehensive understanding of mechanisms of genome functions requires identification of molecules interacting with genomic regions of interest in vivo. We previously developed the insertional chromatin immunoprecipitation (iChIP) technology to isolate specific genomic regions retaining molecular interactions and identify their associated molecules. iChIP consists of locus-tagging and affinity purification. The recognition sequences of an exogenous DNA-binding protein such as LexA are inserted into a genomic region of interest in the cell to be analyzed. The exogenous DNA-binding protein fused with a tag(s) is expressed in the cell and the target genomic region is purified with antibody against the tag(s). In this study, we developed the iChIP system using recombinant DNA-binding proteins to make iChIP more straightforward than the conventional iChIP system using expression of the exogenous DNA-binding proteins in the cells to be analyzed.
\end{abstract}

Results: In this system, recombinant 3xFNLDD-D (r3xFNLDD-D) consisting of the 3xFLAG-tag, a nuclear localization signal (NLS), the DNA-binding domain plus the dimerization domain of the LexA protein, and the Dock-tag is used for isolation of specific genomic regions. r3xFNLDD-D was expressed using a silkworm-baculovirus expression system and purified by affinity purification. iChIP using r3xFNLDD-D could efficiently isolate the single-copy chicken Pax5 (cPax5) locus, in which LexA binding elements were inserted, with negligible contamination of other genomic regions. In addition, we could detect RNA associated with the cPax5 locus using this form of the iChIP system combined with RT-PCR.

Conclusions: The iChIP system using r3xFNLDD-D can isolate specific genomic regions retaining molecular interactions without expression of the exogenous DNA-binding protein in the cell to be analyzed. iChIP using r3xFNLDD-D would be more straightforward and useful for analysis of specific genomic regions to elucidate their functions as compared to the previously published iChIP protocol.

Keywords: iChIP, Locus-specific ChIP, r3xFNLDD-D, ChIP, Chromatin immunoprecipitation

\section{Background}

Genome functions are mediated by various molecular complexes in the context of chromatin [1]. Comprehensive understanding of mechanisms of genome functions requires identification of molecules interacting with genomic regions of interest in vivo. To this end, we recently

\footnotetext{
* Correspondence: hodaka@biken.osaka-u.ac.jp

Chromatin Biochemistry Research Group, Combined Program on Microbiology and Immunology, Research Institute for Microbial Diseases, Osaka University, Suita, Osaka, Japan
}

\section{Biomed Central}

developed the locus-specific chromatin immunoprecipitation (ChIP) technologies consisting of insertional ChIP (iChIP) [2-5] and engineered DNA-binding moleculemediated ChIP (enChIP) [6-8] to isolate genomic regions of interest retaining molecular interactions. The functions of the genomic regions can be comprehensively understood by analysis of DNA, RNA, proteins, or other molecules interacting with the genomic regions.

In principle, iChIP is based on locus-tagging by inserting recognition sequences of an exogenous DNA-binding 
protein to isolate specific genomic regions using the exogenous DNA-binding molecule. In contrast, enChIP is based on recognition of endogenous DNA sequences by engineered DNA-binding molecules such as transactivatorlike (TAL) proteins and the clustered regularly interspaced short palindromic repeats (CRISPR) system. The scheme of iChIP is as follows: (i) The recognition sequences of an exogenous DNA-binding protein such as a bacterial protein, LexA, are inserted into the genomic region of interest in the cell to be analyzed. (ii) The DNA-binding domain (DB) of the exogenous DNA-binding protein is fused with a tag(s) and a nuclear localization signal(s) (NLS(s)) and expressed in the cell to be analyzed. (iii) The resultant cell is stimulated and crosslinked with formaldehyde or other crosslinkers, if necessary. (iv) The cell is lysed, and the chromatin DNA is fragmented by sonication or enzymatic digestion. (v) The complexes including the exogenous DB are immunoprecipitated with antibody $(\mathrm{Ab})$ against the $\operatorname{tag}(\mathrm{s})$. (vi) The isolated complexes which retain molecular interactions are reverse crosslinked, if necessary, and subsequent purification of DNA, RNA, proteins, or other molecules allows their identification and characterization. We successfully identified proteins and RNA components of an insulator, which functions as boundaries of chromatin domains [9], by using iChIP combined with mass spectrometry (iChIP-MS) or RT-PCR (iChIP-RT-PCR) [3]. iChIP has also been used for identification of proteins or DNA interacting with specific genomic regions by other researchers [10-13]. Thus, iChIP is a useful technology for elucidation of molecular mechanisms of genome functions.

We recently developed 3xFNLDD, the second-generation tagged LexA DB consisting of 3xFLAG-tag, an NLS, and DB plus the dimerization domain of LexA, to utilize in iChIP [4]. 3xFNLDD is expressed in the cell to be analyzed for binding to the inserted LexA BE and subsequent purification of target genomic regions in the iChIP technology. If target genomic regions inserted with LexA $\mathrm{BE}$ can be pulled down using recombinant 3xFNLDD conjugated to $\mathrm{Ab}$ against the tag (Figure 1), expression of 3xFNLDD in the cell to be analyzed would not be necessary. In addition, it is not necessary to consider unexpected side effects of expression of 3xFNLDD on cell behavior, if any, making the procedure more straightforward than the conventional iChIP system using expression of the exogenous DNA-binding proteins in the cells to be analyzed.

In this study, we developed the iChIP system using the recombinant C-terminally Dock-tagged 3xFNLDD (r3xFNLDD-D). r3xFNLDD-D was expressed using a silkworm-baculovirus expression system and purified by affinity purification. iChIP using r3xFNLDD-D could effectively isolate the single-copy chicken Pax5 (cPax5) locus from a chicken B cell line, DT40. In addition, we could detect RNA associated with the cPax5 locus using this form of the iChIP system combined with RT-PCR. Thus, iChIP using r3xFNLDD-D would be more straightforward and useful than the conventional iChIP system using expression of the exogenous DNA-binding proteins in the cells to be analyzed to isolate specific genomic regions for their biochemical analysis.

\section{Results and discussion}

\section{Expression and purification of r3xFNLDD-D}

For preparation of the purified r3xFNLDD-D, we utilized a silkworm-baculovirus expression system [14]. In this system, r3xFNLDD-D was expressed in a silkworm pupa by infection of baculoviruses expressing r3xFNLDD-D. The expressed protein was purified from the pupal homogenates using Dock Catch Resin, which specifically binds to Dock-tag in a calcium-dependent manner [14]. As shown in Figure 2A, SDS-PAGE followed by Coomassie Brilliant Blue $(\mathrm{CBB})$ staining detected a single protein band at $35 \mathrm{kDa}$ in the elution fraction. This protein was confirmed as $\mathrm{r} 3 x$ FNLDD-D by immunoblot analysis with anti-Dock $\mathrm{Ab}$ (Figure 2B). Thus, r3xFNLDD-D could be expressed in a silkworm pupa and purified without visible degradation.

\section{Efficient isolation of a target genomic region by iChIP using r3xFNLDD-D}

Next, we examined whether the purified r3xFNLDD-D could be utilized for isolation of genomic regions of interest from vertebrate cells. To this end, we used the chicken DT40-derived cell line, DT40\#205-2, in which $8 \times$ repeats of LexA BE were inserted $0.3 \mathrm{kbp}$ upstream of the exon $1 \mathrm{~A}$ of the single-copy endogenous cPax 5 gene [15] (Figure 3A). The crosslinked chromatin prepared from the cell line was subjected to iChIP using r3xFNLDD-D as shown in Figure 1. After purification of the immunoprecipitated genomic DNA, the yield of the cPax5 1A promoter region was evaluated by detection of the LexA BE site (LexA BE) and the region $0.2 \mathrm{kbp}$ upstream of LexA BE (i.e., $0.7 \mathrm{kbp}$ upstream of the transcription start site (TSS) of cPax5 exon 1A) $(-0.7 \mathrm{k})$ by real-time PCR (Figure 3A). As shown in Figure 3B, the yields of LexA BE and $-0.7 \mathrm{k}$ were more than $20 \%$ and $5 \%$ of input, respectively, when $10 \mu \mathrm{g}$ of each r3xFNLDD-D and anti-FLAG Ab were used. In contrast, the yield of the genomic region $10 \mathrm{kbp}$ upstream of the TSS of the exon 1A $(-10 \mathrm{k})$ was less than $0.01 \%$. These results suggested that r3xFNLDD-D can bind to LexA BE even in the crosslinked chromatin and iChIP using r3xFNLDD-D is able to specifically purify target genomic regions. The specific isolation of the $\mathrm{cPax} 51 \mathrm{~A}$ promoter region was completely blocked when we inhibited binding of r3xFNLDD-D to anti-FLAG Ab with excessive amounts of 3xFLAG peptide (Figure 3C). The cPax5 1A promoter region was not isolated when parental DT40 was used instead of DT40\#205-2 (Figure 3C). These results clearly 


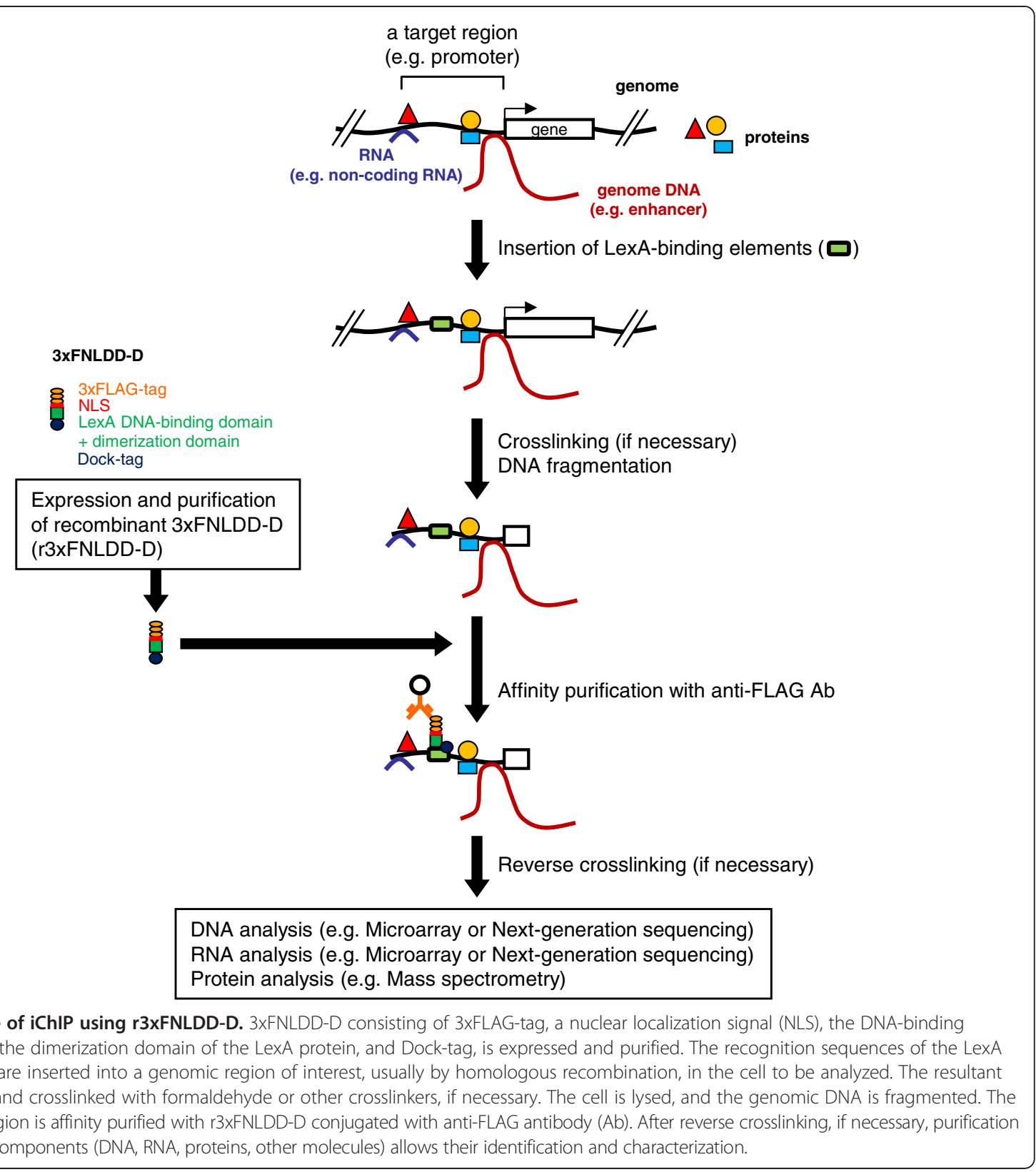

demonstrated that isolation of target genomic regions is mediated by binding of r3xFNLDD-D to LexA BE. The yield of the target genomic region by the modified iChIP system was comparable with that of the previously reported iChIP protocol [4].

\section{Optimization of iChIP using r3xFNLDD-D}

Next, we titrated amounts of r3xFNLDD-D and antiFLAG Ab to optimize the system (Figure 4A). The yield of the LexA BE site in the $\mathrm{cPax5} 1 \mathrm{~A}$ promoter was comparable when $0.5-10 \mu \mathrm{g}$ of each r3xFNLDD-D and anti-FLAG Ab was used with chromatin prepared from $1 \times 10^{7}$ of DT40\#205-2 cells. In contrast, use of $0.01-$
$0.1 \mu \mathrm{g}$ of each protein showed lower yield, suggesting that $0.5 \mu \mathrm{g}$ of each $\mathrm{r} 3 \times$ FNLDD-D and anti-FLAG Ab are sufficient for $1 \times 10^{7}$ cells. The yield of iChIP using $0.5 \mu \mathrm{g}$ of r3xFNLDD-D was $20 \%$ of input for LexA BE and less than $0.01 \%$ for $-10 \mathrm{k}$, which is comparable with that using $10 \mu \mathrm{g}$ of r3xFNLDD-D (Figures $3 \mathrm{~B}$ and $4 \mathrm{~B}$ ). In this regard, we observed the yield of $15 \%$ of input for the same locus when 3xFLNDD was expressed in DT40\#205-2 and the conventional iChIP protocol was used (T.F., H.F., unpublished observation). These results showed that the iChIP using r3xFNLDD-D could purify the target region with efficiency comparable to the conventional iChIP. We also examined whether it would 


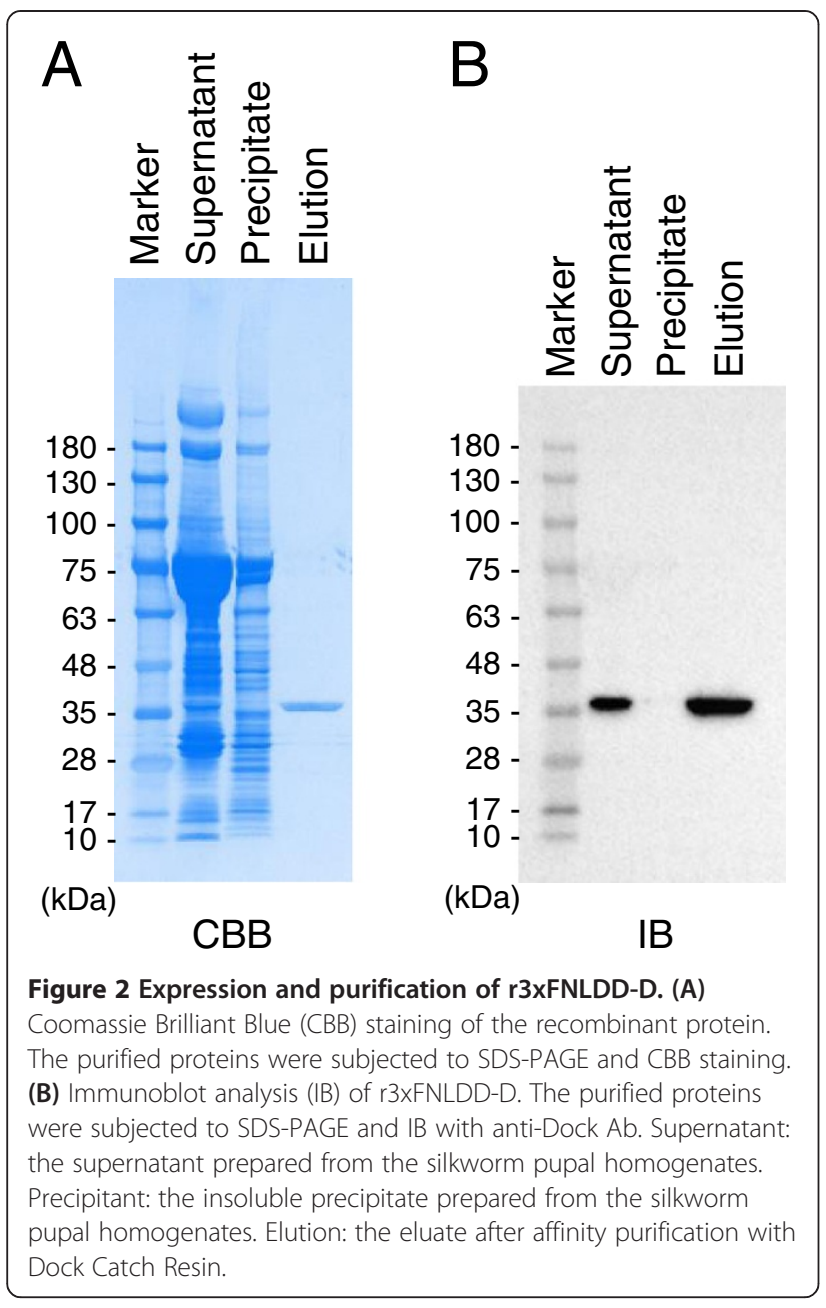

be possible to purify the $\mathrm{cPax} 51 \mathrm{~A}$ promoter region using r3xFNLDD-D with Dock Catch Resin, which binds to the C-terminal Dock-tag of r3xFNLDD-D. As shown in Additional file 1: Figure S1, 2\% of input of the LexA $\mathrm{BE}$ site could be isolated with negligible contamination of $-10 \mathrm{k}$, indicating that the C-terminal Dock-tag of r3xFNLDD-D can also be utilized for iChIP, although the yield was much lower than that using anti-FLAG Ab.

\section{Isolation of RNA associated with the CPax5 locus by iChIP using r3xFNLDD-D}

Next, we examined whether iChIP using r3xFNLDD-D could be utilized to isolate genomic regions of interest and identify molecules interacting with those genomic regions in cells. To this end, we attempted to isolate the cPax5 locus including the exon $1 \mathrm{~A}$ region by iChIP using r3xFNLDD-D and detect the nascent RNA transcribed from the TSS of the cPax5 exon 1A by RT-PCR (Figure 5A). Transcription from the $\mathrm{CPax} 5$ exon $1 \mathrm{~A}$ was not disrupted by the presence of LexA $\mathrm{BE}$ inserted in the $1 \mathrm{~A}$ promoter region (Additional file 1: Figure S2). As shown in Figure 5B, iChIP using r3xFNLDD-D isolated the cPax5 exon $1 \mathrm{~A}$

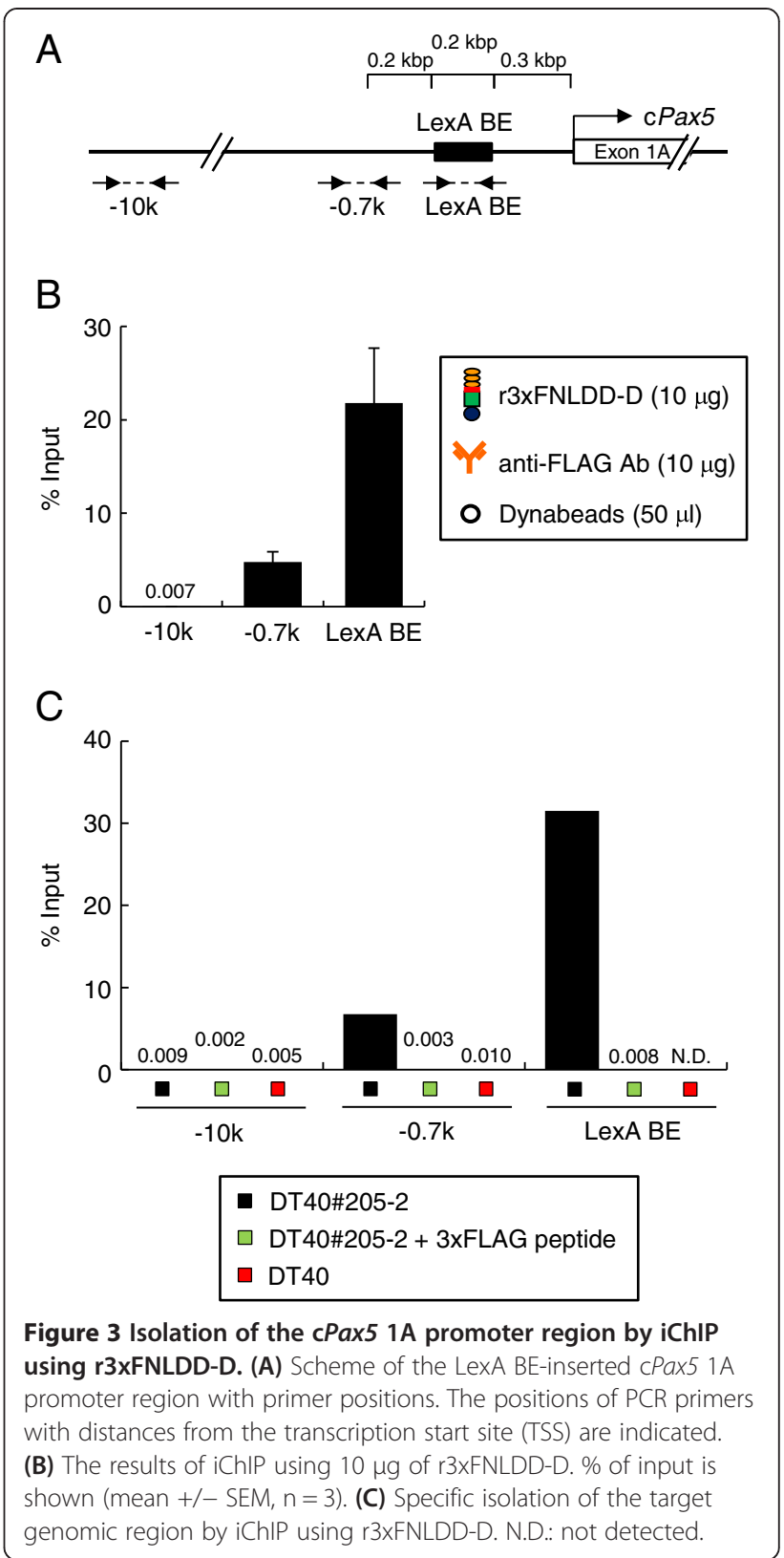

region but not the exon 3 region of the irrelevant cAID gene, which encodes an enzyme essential for B cell-specific immunoglobulin somatic hypermutation and class switch recombination [16]. After purification of the associated RNA, RT-PCR analysis detected RNA transcribed from the exon $1 \mathrm{~A}$ of the cPax5 gene but not that from the cAID gene in the iChIP sample (Figure 5C) (the full-length images with size markers are shown in Additional file 1: Figure S3). These results suggested that iChIP using r3xFNLDD-D is able to isolate specific genomic regions retaining molecules interacting with the genomic regions. 


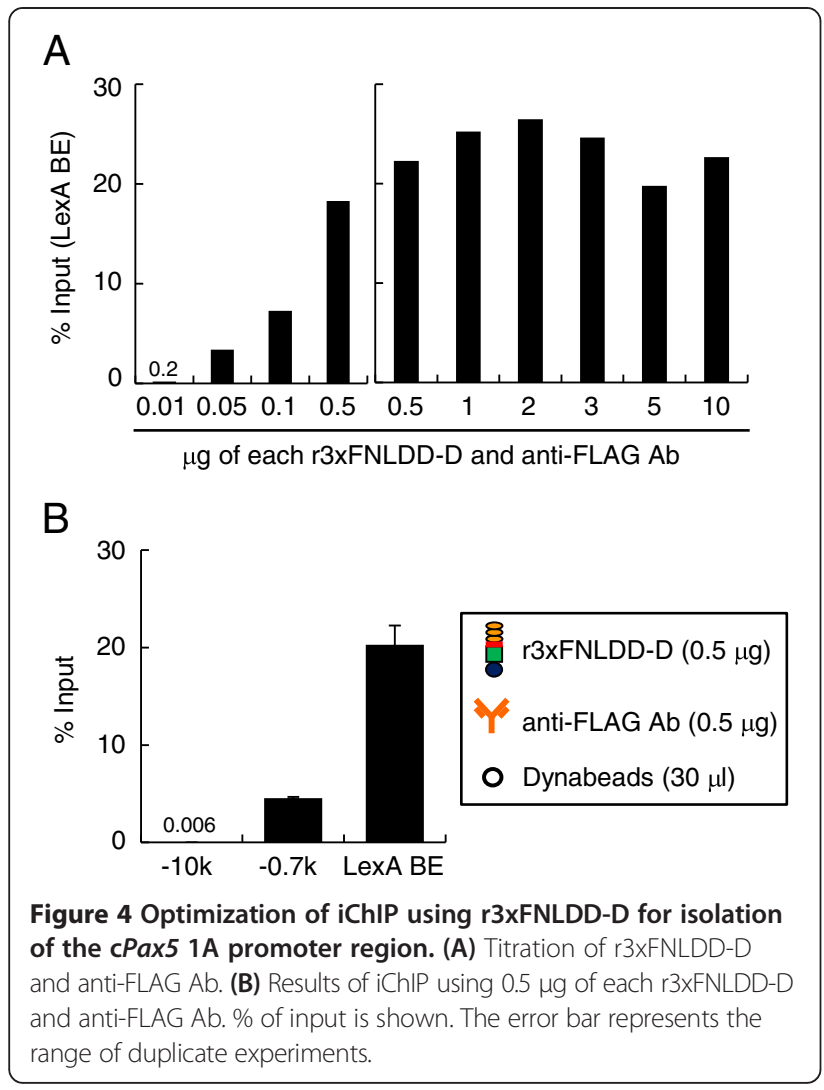

Feasibility of enChIP using recombinant engineered DNA-binding molecules

Lastly, we examined whether enrichment of specific genomic regions is feasible by enChIP using recombinant TAL proteins (Additional file 1: Figure S4). enChIP uses engineered DNA-binding molecules such as TAL proteins or the CRISPR system consisting of a catalytically inactive form of Cas9 (dCas9) and guide RNA (gRNA) for locus-tagging and affinity purification of the target loci [6-8]. We generated a construct expressing a fusion protein, r3xFN-5'HS5-TAL-G, consisting of 3xFLAG-tag, an NLS, a recombinant TAL protein recognizing human 5' HS5 region, which functions as an insulator to regulate transcription of the $\beta$-globin genes (Additional file 1 : Figure S5A) [17], and the glutathione S-transferase (GST)-tag, and prepared the recombinant protein by using the silkworm-baculovirus expression system (Additional file 1: Figure $\mathrm{S} 5 \mathrm{~B}$ and $\mathrm{C}$ ). We used the recombinant protein for enChIP analysis of the 5'HS5 locus. As shown in Additional file 1: Figure S5D, the 5'HS5 site was enriched several-fold compared to the irrelevant interferon regulatory factor 1 (IRF-1) promoter region when non-crosslinked native chromatin prepared from the 293T cell line was used. These results suggest that enChIP using recombinant TAL proteins is feasible. However, we found that the r3xFN-5'HS5-TAL-G (ca. $160 \mathrm{kDa}$ ) showed massive degradation (Additional file 1:

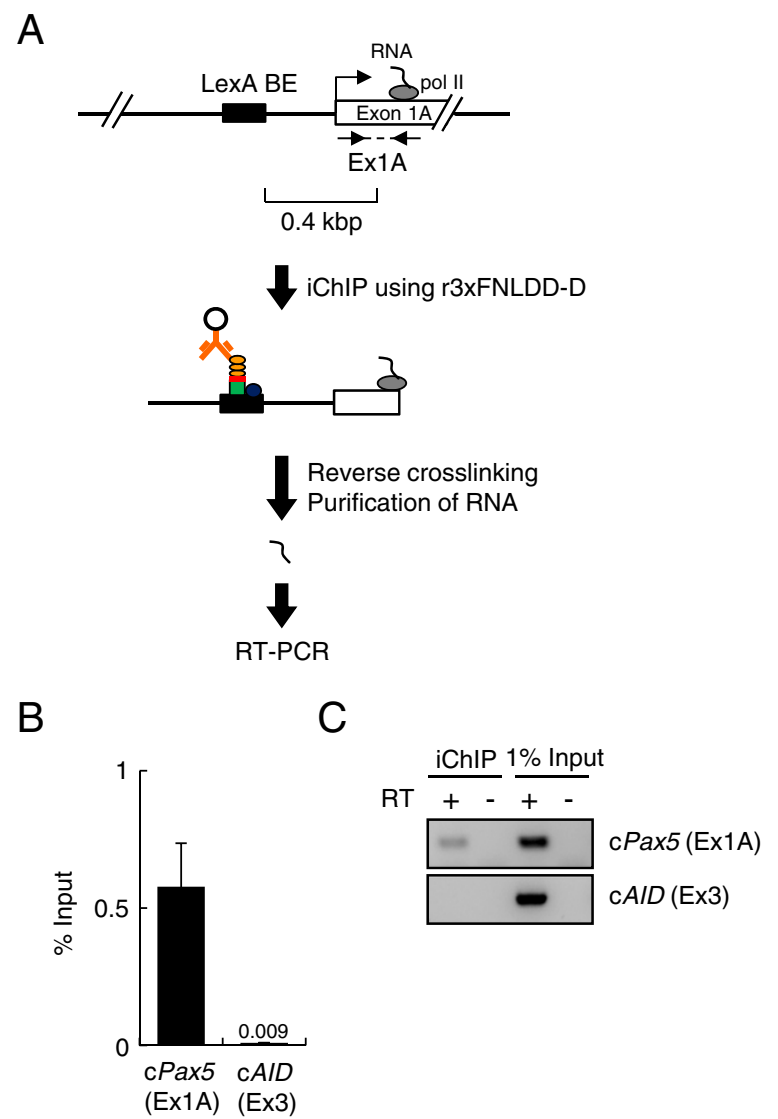

Figure 5 Detection of RNA associated with the cPax 5 locus.

(A) Scheme of iChIP. After isolation of the CPax5 locus by iChIP using r3xFNLDD-D, the nascent RNA transcribed on the exon 1A of the CPax5 gene was detected by RT-PCR. (B) Results of iChIP using $0.5 \mu \mathrm{g}$ of each r3xFNLDD-D and anti-FLAG Ab. \% of input is shown. The error bar represents the range of duplicate experiments. (C) Detection of RNA corresponding to the exon $1 \mathrm{~A}$ of the $\mathrm{CPax} 5$ gene but not that corresponding to the exon 3 of the CAID gene by RT-PCR.

Figure S5B and C). In addition, we failed to detect enrichment of the target 5'HS5 site when we used crosslinked chromatin (data not shown). These results also suggest that improvement in production of recombinant TAL proteins and their access to target loci might be required for efficient isolation of target regions and identification of associated molecules.

\section{Applications and advantages of iChIP using r3xFNLDD-D} In this study, we applied RT-PCR to detection of RNA interacting with a genomic region of interest in cells. Next-generation sequencing or microarray analysis can be combined with iChIP using r3xFNLDD-D for nonbiased identification of interacting RNA as well as DNA. Moreover, mass spectrometry can be combined for nonbiased identification of interacting proteins.

Because iChIP using r3xFNLDD-D does not require expression of 3xFNLDD in cells, it is of great use in the 
iChIP analysis of primary cells isolated from organisms, especially higher eukaryotes such as mice. In the case of application of the standard iChIP technology to mice, it is time-consuming to establish mouse lines expressing 3xFNLDD in the cells to be analyzed as well as those possessing LexA BE in specific genomic regions. In this regard, iChIP using r3xFNLDD-D is able to skip the mating steps between mice expressing 3xFNLDD and those possessing LexA BE, substantially accelerating iChIP analysis using organisms.

Compared to enChIP or proteomics of isolated chromatin (PICh), which uses specific biotinylated nucleic acid probes such as locked nucleic acids (LNAs) that hybridize target genomic regions for their isolation [18], iChIP requires insertion of LexA $\mathrm{BE}$, which takes time and effort. However, recent advancement of genome editing technologies using TALEN and CRISPR makes insertion of exogenous sequences in target loci much more easily. In addition, insertion of such exogenous sequences may abrogate function of genomic regions through changes in nucleosome positioning or other mechanisms. Therefore, it is necessary to confirm that the insertion of LexA BE does not abrogate function of genomic regions before isolating the genomic regions by iChIP. On the other hand, the locus-tagging system used in iChIP can be used for isolation of a specific target allele such as a maternal or paternal allele. Feasibility of such allele-specific analysis is one of advantages of iChIP over enChIP and PICh when allele-specific analysis is required, for example, in the analysis of genome imprinting.

\section{Conclusions}

In this study, we established the iChIP system using r3xFNLDD-D to make the iChIP technology much more straightforward than the conventional iChIP system using expression of the exogenous DNA-binding proteins in the cells to be analyzed. Using this system, we were able to isolate target genomic regions; \% of input reached more than $20 \%$ for the cPax 1 A promoter region. In addition, we could detect RNA associated with the cPax5 locus, suggesting that iChIP using r3xFNLDD-D can isolate target genomic regions retaining molecular interactions. Thus, the modified iChIP protocol described here using r3xFNLDD-D has advantages over the previously published protocol in that it is more straightforward and useful for analysis of specific genomic regions to elucidate their functions.

\section{Methods}

\section{Expression and purification of r3xFNLDD-D}

Expression of 3xFNLDD-D was performed using the silkworm-baculovirus expression system (ProCube) (Sysmex Corporation, http://procube.sysmex.co.jp/eng/) as described previously [14]. Briefly, the coding sequence of 3xFNLDD [4] was inserted into the transfer vector pM31a (Sysmex Corporation) to fuse the Dock-tag at its C-terminus and co-transfected with linearized genomic DNA of ABv baculovirus (Bombyx mori nucleopolyhedrovirus; CPd strain, Sysmex Corporation) into the B. mori-derived cell line, $\mathrm{BmN}$, to generate the recombinant baculovirus. The generated baculovirus was infected into a silkworm pupa to express 3xFNLDD-D. The expressed 3xFNLDD-D was

Table 1 Primers used in this study

\begin{tabular}{|c|c|c|c|}
\hline Number & Name & Sequence $\left(5^{\prime} \rightarrow 3^{\prime}\right)$ & Experiments \\
\hline 26572 & LexA-N2 & ttctctatcgataggtacctcg & Real-time PCR in Figures 3, 4 and Additional file 1: Figure S1 (LexA BE) \\
\hline 27428 & LexA-C-for-Pax5 & cgctgcgtggtcgagcgtactg & Real-time PCR in Figures 3, 4 and Additional file 1: Figure S1 (LexA BE) \\
\hline 27134 & CPax5-ChIP-UP(-0.2 k)-F & gggctcttatttcgtttttcttgtt & Real-time PCR in Figures 3 and 4 (-0.7 k) \\
\hline 27135 & CPax5-ChIP-UP(-0.2 k)-R & gtgcttatttgtcagcgtggttg & Real-time PCR in Figures 3 and 4 (-0.7 k) \\
\hline 27013 & CPax5-ChIP-UP(-10 k)-F & tccacatcgttacattgtcacttct & Real-time PCR in Figures 3, 4 and Additional file 1: Figure S1 (-10 k) \\
\hline 27014 & CPax5-ChIP-UP(-10 k)-R & taaaagccctcagttcgatttattg & Real-time PCR in Figures 3, 4 and Additional file 1: Figure S1 (-10 k) \\
\hline 26552 & cPax5-inExon1A-F & cctaaaacgtttagtttcagctcagt & RT-PCR in Figure 5 and Additional file 1: Figure S2 (CPax5 Ex1A) \\
\hline 26553 & cPax5-inExon1A-R & ttcgtggctctctcaggtca & RT-PCR in Figure 5 and Additional file 1: Figure S2 (cPax5 Ex1A) \\
\hline 27571 & CAID-Ex3-F & catgtggaggttctcttcctacg & RT-PCR in Figure 5 and Additional file 1: Figure S2 (CAID Ex3) \\
\hline 27572 & CAID-Ex3-R & caagtttgggtaggcacgaag & RT-PCR in Figure 5 and Additional file 1: Figure S2 (CAID Ex3) \\
\hline 26773 & 18SrRNA-F2 & cttagagggacaagtggcg & RT-PCR in Additional file 1: Figure S2 (18S) \\
\hline 26774 & 18SrRNA-R2 & acgctgagccagtcagtgta & RT-PCR in Additional file 1: Figure S2 (18S) \\
\hline 27420 & hHS5-TAL-Target-F & ccagtttctccagtttccctttt & Real-time PCR in Additional file 1: Figure S5 (5'HS5) \\
\hline 27421 & hHS5-TAL-Target-R & ttttcaaaatgcaaggtgatgtc & Real-time PCR in Additional file 1: Figure S5 (5'HS5) \\
\hline 27310 & hIRF1-prom-F & cgcctgcgttcgggagatatac & Real-time PCR in Additional file 1: Figure S5 (IRF-1) \\
\hline 27312 & hIRF1-prom-R1 + 2 & ctgtcctctcactccgecttgt & Real-time PCR in Additional file 1: Figure S5 (IRF-1) \\
\hline
\end{tabular}


purified with Dock Catch Resin (Sysmex Corporation) as described previously [14]. The immunoblot analysis was performed with anti-Dock Ab (Sysmex Corporation).

\section{Cell lines}

The chicken B cell line DT40 was provided by the RIKEN BioResource Center through the National Bio-Resource Project of the Ministry of Education, Science, Sports and Culture of Japan. DT40 and DT40\#205-2, in which LexA $\mathrm{BE}$ was inserted in the $1 \mathrm{~A}$ promoter region of the $\mathrm{cPax5}$ gene (Fujita and Fujii, manuscript submitted), were maintained in RPMI-1640 (Wako) with $4 \mathrm{mM}$ glutamine, 10\% $(\mathrm{v} / \mathrm{v})$ fetal bovine serum, $1 \%$ chicken serum, and $50 \mu \mathrm{M}$ 2-mercaptoethanol at $39.5^{\circ} \mathrm{C}$.

\section{Chromatin preparation}

Cells $\left(2 \times 10^{7}\right)$ were fixed with $1 \%$ formaldehyde at $37^{\circ} \mathrm{C}$ for $5 \mathrm{~min}$. The chromatin fraction was extracted and fragmented ( $2 \mathrm{kbp}$-long on average) by sonication as described previously [19] except for using $800 \mu \mathrm{l}$ of in vitro Modified Lysis Buffer 3 (10 mM Tris pH 8.0, $150 \mathrm{mM} \mathrm{NaCl}, 1 \mathrm{mM}$ EDTA, $0.5 \mathrm{mM}$ EGTA) and Ultrasonic disruptor UD-201 (TOMY SEIKO). After sonication, Triton X-100 was added to final concentration at $0.1 \%$.

\section{iChIP using r3xFNLDD-D}

The sonicated chromatin $(400 \mu \mathrm{l})$ was pre-cleared with $0.01-10 \mu \mathrm{g}$ of normal mouse IgG (Santa Cruz Biotechnology) conjugated to 30 - $50 \mu \mathrm{l}$ of Dynabeads-Protein G (Invitrogen) and subsequently incubated with $0.01-10 \mu \mathrm{g}$ of r3xFNLDD-D and anti-FLAG M2 Ab (Sigma-Aldrich) conjugated to $30-50 \mu \mathrm{l}$ of Dynabeads-Protein $\mathrm{G}$ at $4^{\circ} \mathrm{C}$ for $20 \mathrm{~h} .100 \mu \mathrm{g}$ of 3xFLAG peptide was added to inhibit binding of r3xFNLDD-D to anti-FLAG Ab. The Dynabeads were washed four times with $1 \mathrm{ml}$ of in vitro Wash Buffer (20 mM Tris pH 8.0, $150 \mathrm{mM} \mathrm{NaCl}, 2$ mM EDTA, $0.1 \%$ Triton X-100) and once with $1 \mathrm{ml}$ of TBS-IGEPALCA630 (50 mM Tris pH 7.5, $150 \mathrm{mM} \mathrm{NaCl}$, 0.1\% IGEPALCA630). The isolated chromatin complexes were eluted with $120 \mu \mathrm{l}$ of Elution Buffer $(500 \mu \mathrm{g} / \mathrm{ml}$ 3xFLAG peptide (Sigma-Aldrich), $50 \mathrm{mM}$ Tris pH 7.5, $150 \mathrm{mM} \mathrm{NaCl}, 0.1 \%$ IGEPAL-CA630) at $37^{\circ} \mathrm{C}$ for $30 \mathrm{~min}$. After reverse crosslinking at $65^{\circ} \mathrm{C}$, DNA was purified with ChIP DNA Clean \& Concentrator (Zymo Research) and used as template for real-time PCR with SYBR Select PCR system (Applied Biosystems) using the Applied Biosystems 7900HT Fast Real-Time PCR System. PCR cycles were as follows: heating at $50^{\circ} \mathrm{C}$ for $2 \mathrm{~min}$ followed by $95^{\circ} \mathrm{C}$ for $10 \mathrm{~min} ; 40$ cycles of $95^{\circ} \mathrm{C}$ for $15 \mathrm{sec}$ and $60^{\circ} \mathrm{C}$ for $1 \mathrm{~min}$. The primers used in this experiment are shown in Table 1.

\section{Isolation of interacting RNA and RT-PCR}

Chromatin preparation and iChIP using r3xFNLDD-D were performed as described above except for addition of RNasin Plus RNase Inhibitor (Promega) in all buffers. After reverse crosslinking at $65^{\circ} \mathrm{C}$, RNA was isolated with Isogen II (Nippon gene) combined with Direct-zol RNA Mini Prep (Zymo Research). The purified RNA was used as template for reverse transcription with ReverTra Ace qPCR RT Master Mix with gDNA Remover (Toyobo). The cDNA was used as template for PCR with AmpliTaq Gold 360 Master Mix (Applied Biosystems). PCR cycles were as follows: heating at $95^{\circ} \mathrm{C}$ for $10 \mathrm{~min} ; 40$ cycles of $95^{\circ} \mathrm{C}$ for $30 \mathrm{sec}, 60^{\circ} \mathrm{C}$ for $30 \mathrm{sec}, 72^{\circ} \mathrm{C}$ for $1 \mathrm{~min}$; and the final extending $72^{\circ} \mathrm{C}$ for $2 \mathrm{~min}$. The primers used in this experiment are shown in Table 1.

\section{Additional file}

Additional file 1: Supplemental Materials.

\section{Competing interests}

Patents on iChIP are already registered ("Method for isolating specific genomic regions", August 20, 2010, PCT/JP2010/064052; April 9, 2013, US patent 8415098; November 22, 2013, Japanese patent 5413924). The authors filed a patent application on enChIP ("Method for isolating specific genomic regions using DNA-binding molecules recognizing endogenous DNA sequences", September 6, 2013, PCT/JP2013/74107). This does not alter the authors' adherence to all the BMC Molecular Biology policies on sharing data and materials.

\section{Authors' contributions}

TF and HF conceived this form of the iChIP and enChIP technologies, designed and performed experiments, and wrote the manuscript. HF directed and supervised the study. Both authors read and approved the final manuscript.

\section{Acknowledgements}

We thank F. Kitaura for technical assistance. This work was supported by Takeda Science Foundation (TF), the Uehara Memorial Foundation (HF), the Kurata Memorial Hitachi Science and Technology Foundation (TF and HF), Adaptable \& Seamless Technology Transfer Program through Target-driven R\&D (A-STEP) by the Japan Science and Technology Agency (JST) (\#AS251Z01861Q) (HF), Grant-in-Aid for Young Scientists (B) (\#25830131) (TF), "Transcription Cycle" (\#25118512) (HF) from the Ministry of Education, Culture, Sports, Science and Technology of Japan.

Received: 13 May 2014 Accepted: 12 November 2014 Published online: 27 November 2014

\section{References}

1. van Driel R, Fransz PF, Verschure PJ: The eukaryotic genome: a system regulated at different hierarchical levels. J Cell Sci 2003, 116:4067-4075.

2. Hoshino A, Fujii H: Insertional chromatin immunoprecipitation: a method for isolating specific genomic regions. J Biosci Bioeng 2009, 108(5):446-449.

3. Fujita T, Fujii H: Direct idenification of insulator components by insertional chromatin immunoprecipitation. PLoS One 2011, 6(10):e26109.

4. Fujita T, Fujii H: Efficient isolation of specific genomic regions by insertional chromatin immunoprecipitation (iChIP) with a second-generation tagged LexA DNA-binding domain. Adv Biosci Biotechnol 2012, 3(5):626-629.

5. Fujita T, Fujii H: Locus-specific biochemical epigenetics/chromatin biochemistry by insertional chromatin immunoprecipitation. ISRN Biochem 2013, 2013:913273.

6. Fujita T, Fujii $\mathrm{H}$ : Efficient isolation of specific genomic regions and identification of associated proteins by engineered DNA-binding molecule-mediated chromatin immunoprecipitation (enChIP) using CRISPR. Biochem Biophys Res Commun 2013, 439:132-136. 
7. Fujita T, Asano Y, Ohtsuka J, Takada Y, Saito K, Ohki R, Fujii H: Identification of telomere-associated molecules by engineered DNA-binding molecule-mediated chromatin immunoprecipitation (enChIP). Sci Rep 2013, 3:3171.

8. Fujita T, Fujii H: Identification of proteins associated with an IFNy-responsive promoter by a retroviral expression system for enChIP using CRISPR. PLoS One 2014, 9(7):e103084.

9. Gaszner M, Felsenfeld G: Insulators: exploiting transcriptional and epigenetic mechanisms. Nat Rev Genet 2006, 7:703-713.

10. McCullagh E, Seshan A, El-Samad H, Madhani HD: Coordinate control of gene expression noise and interchromosomal interactions in a MAP kinase pathway. Nat Cell Biol 2010, 12(10):954-962.

11. Agelopoulos M, McKay DJ, Mann RS: Developmental regulation of chromain conformation by Hox proteins in Drosophila. Cell Rep 2012, 1(4):350-359.

12. Byrum SD, Raman A, Taverna SD, Tackett A: ChAP-MS: a method for identification of proteins and histone posttranslational modifications at a single genomic locus. Cell Rep 2012, 2(1):198-205.

13. Pourfarzad F, Aghajanirefah A, de Boer E, Ten Have S, Bryn van Dijk T, Kheradmandkia S, Stadhouders R, Thongjuea S, Soler E, Gillemans N, von Lindern M, Demmers J, Philipsen S, Grosveld F: Locus-specific proteomics by TChP: targeted chromatin purification. Cell Rep 2013, 4:589-600.

14. Kamezaki Y, Enomoto C, Ishikawa Y, Koyama T, Nara S, Suzuki T, Sakka K: The Dock tag, an affinity tool for the purification of recombinant proteins, based on the interaction between dockerin and cohesin comains from Clostridium josui cellulosome. Protein Expr Purif 2010, 70:23-31.

15. Fujita T, Fujii H: Species-specific 5 '-genomic structure and multiple transcription start sites in the chicken Pax5 gene. Gene 2011, 477:24-31.

16. Arakawa $\mathrm{H}$, Buerstedde JM: Immunoglobulin gene conversion: insights from bursal B cells and the DT40 cell line. Dev Dyn 2004, 229:458-464.

17. Kukreti S, Kaur H, Kaushik M, Bansal A, Saxena S, Kaushik S, Kukreti R: Structural polymorphism at LCR and its role in beta-globin gene regulation. Biochimie 2010, 92:1199-1206.

18. Déjardin J, Kingston RE: Purification of proteins associated with specific genomic loci. Cell 2009, 136(1):175-186.

19. Fuijta T, Ryser S, Tortola S, Piuz I, Schlegel W: Gene-specific recruitment of positive and negative elongation factors during stimulated transcription of the MKP-1 gene in neuroendocrine cells. Nucleic Acids Res 2007, 35:1007-1017.

doi:10.1186/s12867-014-0026-0

Cite this article as: Fujita and Fujii: Efficient isolation of specific genomic regions retaining molecular interactions by the iChIP system using recombinant exogenous DNA-binding proteins. BMC Molecular Biology 2014 15:26.

\section{Submit your next manuscript to BioMed Central and take full advantage of:}

- Convenient online submission

- Thorough peer review

- No space constraints or color figure charges

- Immediate publication on acceptance

- Inclusion in PubMed, CAS, Scopus and Google Scholar

- Research which is freely available for redistribution 